\title{
OPTIMAL HITTING TIME AND PERPETUAL OPTION IN A NON-LÉVY MODEL: APPLICATION TO REAL OPTIONS
}

\author{
P. BARRIEU, ${ }^{*}$ London School of Economics \\ N. BELLAMY,** Université d'Evry Val d'Essonne
}

\begin{abstract}
We study the perpetual American option characteristics in the case where the underlying dynamics involve a Brownian motion and a point process with a stochastic intensity. No assumption on the distribution of the jump size is made and we work with an arbitrary positive or negative jump. After proving the existence of an optimal stopping time for the problem and characterizing it as the hitting time of an optimal boundary, we provide closed-form formulae for the option value, as well as for the Laplace transform of the optimal stopping time. These results are then applied to the analysis of a real option problem when considering the impact of a fundamental and brutal change in the investment project environment. The consequences of this impact, that can seriously modify, positively or negatively, the project's future cash flows and therefore the investment decision, are illustrated by numerical examples.
\end{abstract}

Keywords: Optimal stopping time; perpetual American option; point process; real option 2000 Mathematics Subject Classification: Primary 60G40; 60G55

Secondary 60K99; 91B28

\section{Introduction}

In this paper, we consider a stochastic process $S$ with dynamics involving a diffusion and a unique jump process. In such a framework, we aim at studying the problem

$$
\underset{\tau \in \Upsilon_{t}}{\operatorname{ess} \sup } \mathrm{E}\left(\mathrm{e}^{-\mu(\tau-t)}\left(S_{\tau}-1\right)^{+} \mid \mathscr{F}_{t}\right),
$$

where $\left(\mathcal{F}_{t}\right)$ is the considered filtration, which will be made precise later, $\Upsilon_{t}$ denotes the set of $\mathcal{F}_{t}$-stopping times taking values in $\left(t,+\infty\left[,(\cdot)^{+}\right.\right.$denotes the positive part, and $\mu$ is a positive real number. This issue comes from real options and, more precisely, from the investor's problem to finance a project, when a (unique) sudden and drastic change can occur in the environment. No assumption is made on the impact of this shock. As a consequence, even if our study includes credit risk issues, it cannot be reduced to this type of problem. In particular, we can also consider positive consequences of the drastic change on the project.

Real options studies are usually written in a continuous framework for the underlying dynamics (see the seminal papers by Brennan and Schwartz (1985), McDonald and Siegel (1986), and Dixit and Pindyck (1993)). But the existence of crises and shocks on investment

Received 14 June 2005; revision received 27 March 2007.

* Postal address: Department of Statistics, London School of Economics, Houghton Street, London WC2A 2AE, UK. Email address: p.m.barrieu@1se.ac.uk

** Postal address: Equipe d'Analyse et Probabilités, Université d'Evry Val d'Essonne, Rue du Père Jarlan, 91025 Evry Cedex, France. Email address: nadine.bellamy@univ-evry.fr 
markets generates discontinuities. Some research has been undertaken studying the impact of such instabilities on the investment decision process (see, for example, Gerber and Shiu (1998), Mordecki (2002), or Barrieu and Bellamy (2005)).

The question of the impact of a brutal change in the project environment has not been studied so far, to our knowledge. Such a fundamental change in the regulation can seriously impact, positively or negatively, the project's future cash flows and therefore the investment decision. Many situations can lead to a major brutal change in the project environment; for instance, a currency devaluation, an increase in interest rates, the appearance of brand-new technologies, or the entry of a new competing firm. Mathematically speaking, all these situations are similar and are equivalent to considering a single jump diffusion process.

This work aims at studying the impact of such a market perturbation on the decision taking. More precisely, the investor has a budget allocation problem. He has to determine whether the project is interesting and if so, to have some idea of the optimal investment time to allocate his capital among financial instruments with different maturities. Therefore, we need to specify the optimal benefit/cost ratio, the project investment value, and the optimal time to enter the project for the investor when he considers the project. This problem is similar to the study of a perpetual American call option written on the project ratio with strike 1 . However, since this paper aims at analyzing a real option problem, we do not adopt a risk neutral logic, and the calculations are made with respect to the prior probability measure, without inducing any loss of generality.

In Section 2, we introduce the framework of study and present our model. The relationship between a real option problem and the pricing of a perpetual American option is also recalled. The main results on optimal stopping and perpetual options characteristics are given in Section 3 . We obtain in this section closed-form formulae without any assumption on the distribution of the jump size and with an arbitrary positive or negative jump. Section 4 is devoted to the study of real option problems in a disrupted framework. We first illustrate the main results of this paper with different investment examples. Then we analyze the importance of the calibration of the model and study the consequences of a misspecification in a bearish environment. Finally, some proofs are presented in Appendix A.

\section{The model}

\subsection{Framework}

In this paper, we consider a given economic agent having the opportunity to invest in a particular project. The stochastic framework is described by a probability space $(\Omega, \mathcal{F}, P)$ where $\mathrm{P}$ is the prior probability measure representing the beliefs and anticipations of the agent.

The project is characterized at any time $t \geq 0$ by its profit/cost ratio $S_{t}$, with

$$
\begin{aligned}
\mathrm{d} S_{t} & =S_{t^{-}}\left(\alpha \mathrm{d} t+\sigma \mathrm{d} W_{t}+\Phi \mathrm{d} D_{t}\right), \\
S_{0} & =s_{0},
\end{aligned}
$$

where the following statements hold.

- Let $\left(W_{t} ; t \geq 0\right)$ be a P-Brownian motion and let $\alpha$ and $\sigma$ be constants.

- The process $\left(D_{t} ; t \geq 0\right)$ describes the potential shock of a drastic change in regulation on the project. It is defined as

$$
D_{t}=\mathbf{1}_{\{t \geq T\}},
$$


where $T$ is a random variable of exponential law with parameter $\lambda$ and $\mathbf{1}_{\{\cdot\}}$ denotes the indicator function. We denote the compensated martingale associated with $\left(D_{t} ; t \geq 0\right)$ by $\left(M_{t} ; t \geq 0\right)$, where

$$
M_{t}=D_{t}-\lambda \int_{0}^{t}\left(1-D_{s}\right) \mathrm{d} s .
$$

- The jump size $\Phi$ is a random variable. More precisely, $\Phi$ measures the impact of the perturbation on the profit/cost ratio.

Therefore, the solution of the stochastic differential equation (1) is

$$
S_{t}=s_{0}(1+\Phi)^{D_{t}} \exp \left[\left(\alpha-\frac{1}{2} \sigma^{2}\right) t+\sigma W_{t}\right]
$$

We assume that the Brownian motion and the random variable $T$ are independent. Moreover, the random variable $\Phi$ is taken independent of both the Brownian motion and the jump time process. Note that such an assumption is reasonable since the (regulation) shock is exogenous to the project we consider.

Assumption 1. From now on, we make the following technical assumptions, ensuring that the problem we consider is well-defined and nondegenerate:

(i) $-1<\underline{\Phi} \leq \Phi<\bar{\Phi}$, for two constants $\underline{\Phi}$ and $\bar{\Phi}$,

(ii) $0<s_{0}<1$,

(iii) $\sigma>0$,

(iv) $\mu>\max (0, \alpha, \alpha+\lambda \bar{\Phi})$.

In Assumption 1(i), $\Phi>-1$ implies that the ratio $S$ remains strictly positive, therefore the investment opportunity is never worthless. Considering a bounded $\Phi$ ensures that the positive impact of a drastic change in the environment cannot be infinite. Therefore, the situation we study is neither catastrophic nor explosive; $\Phi$ is also assumed to be not identically equal to zero, otherwise this study is reduced to the standard Black-Scholes framework.

Assumption 1(ii) states that the project is interesting $\left(s_{0}>0\right)$ but also that the problem is about a 'true' decision since $s_{0}$ is (strictly) less than one; delaying the project realization is only relevant in the case where the profits/costs ratio is less than one.

Assumption 1(iii) maintains a Brownian source of randomness in the process dynamics. It is indeed reasonable to assume that the ratio profits/costs randomly evolves even without a major modification of the project environment. Assumption 1(iv) is an integrability condition.

The available information structure is characterized by the filtration $\left(\mathcal{F}_{t} ; t \geq 0\right)$. At each instant of time $t$, the agent knows what is the current value of the ratio profit/cost related to the project, and therefore knows that $\sigma\left(S_{s} ; 0 \leq s \leq t\right)$. This information includes the value of $D_{t}$; indeed, at each instant $t$, from the observation of the underlying process $S$, the agent knows whether the shock has already occurred or not. As a consequence, the filtration $\mathcal{F}_{t}$ is defined as

$$
\mathcal{F}_{t}=\sigma\left(S_{s}, D_{s} ; 0 \leq s \leq t\right)
$$

Note that the approach defined by the framework we consider differs from those of the models in the literature related to both real options and American options. On the contrary to most of the existing literature (see for instance Gerber and Shiu (1998), Mordecki (2002), or Chesney and 
Jeanblanc (2004)), the underlying process $S$ cannot be written in terms of a Lévy process $X_{t}$ as $S_{0} \exp \left(X_{t}\right)$. Quite recently, Muroi (2002) considered a general framework involving a possible default for the underlying process of an American option. His approach is however different in the sense that it is focused on the numerical aspects and based upon partial differential equation methods. Moreover he does not provide closed-form characterizations for the American option.

\subsection{Perpetual option and investment problem}

The investor has neither any obligation to undertake it nor any time constraint to take his decision. Therefore, the investment decision problem is often brought down to the study of a perpetual American option with the profit/cost ratio $S$ as underlying and 1 as striking level (see, for example, McDonald and Siegel (1986)). Solving the real option problem at any time $t$ is equivalent to determine the value of the quantity

$$
C_{t}=\underset{\tau \in \Upsilon_{t}}{\operatorname{ess} \sup } \operatorname{E}\left(\mathrm{e}^{-\mu(\tau-t)}\left(S_{\tau}-1\right)^{+} \mid \mathcal{F}_{t}\right)
$$

where $\Upsilon_{t}$ denotes the set of $\widetilde{F}_{t}$-stopping times taking values in $(t,+\infty$ [ and where $\mu$ is the discount rate, which can be different from the instantaneous risk-free rate and represents the agent's preferences for the present. Also, E is the expectation with respect to the prior probability measure $\mathrm{P}$.

Since the pair $(D, S)$, where $D$ is defined in (2), is Markovian, we can rewrite $C_{t}$ as

$$
\begin{aligned}
C_{t} & =\underset{\tau \in \Upsilon_{t}}{\operatorname{ess} \sup } \mathrm{E}\left(\mathrm{e}^{-\mu(\tau-t)}\left(S_{\tau}-1\right)^{+} \mid \mathcal{F}_{t}\right) \\
& =\underset{\tau \in \Upsilon_{t}}{\operatorname{ess} \sup } \mathrm{E}\left(\mathrm{e}^{-\mu(\tau-t)}\left(S_{\tau}-1\right)^{+} \mid S_{t}, D_{t}\right),
\end{aligned}
$$

or for all $(t, x, d) \in \mathbb{R}^{+} \times \mathbb{R}^{+} \times\{0,1\}$, and adopting the natural notation,we obtain

$$
C(t, x, d)=\underset{\tau \in \Upsilon_{t}}{\operatorname{ess} \sup } \mathrm{E}\left(\mathrm{e}^{-\mu(\tau-t)}\left(S_{\tau}-1\right)^{+} \mid S_{t}=x, D_{t}=d\right) .
$$

Note that the computations are made with respect to the prior probability measure $\mathrm{P}$, corresponding to the agent's beliefs. This does not create any loss of generality. If the underlying asset of the investment option is traded on financial market, a risk-neutral valuation formula can be easily obtained by considering a risk-neutral probability measure, as underlined in the following remark.

Remark 1. If a risk-neutral approach is adopted, then the only changes in the results come from both the modification of the discount rate $\mu$ in the instantaneous risk-free rate $r$, and a modification in the jump process intensity. More precisely, any equivalent martingale measure $\mathrm{P}^{(\psi, \gamma)}$ is defined in terms of a pair of processes $(\psi, \gamma)$, satisfying the usual regularity conditions and being the solution to

$$
\begin{aligned}
& \alpha+\lambda \Phi\left(1-D_{t}\right)-r+\sigma \psi_{t}+\lambda \Phi \gamma_{t}=0, \\
& \gamma_{t}>-1
\end{aligned}
$$

The Radon-Nikodym derivative

$$
\left.\frac{\mathrm{dP}^{(\psi, \gamma)}}{\mathrm{dP}}\right|_{\mathcal{F}_{t}}=L_{t}^{(\psi, \gamma)}
$$


is given by

$$
\mathrm{d} L_{t}^{(\psi, \gamma)}=L_{t}^{(\psi, \gamma)}\left(\psi_{t} \mathrm{~d} W_{t}+\gamma_{t} \mathrm{~d} M_{t}\right), \quad L_{0}^{(\psi, \gamma)}=1 .
$$

Therefore, the intensity of $\left(D_{t} ; t \geq 0\right)$ evaluated with respect to $\mathrm{P}^{(\psi, \gamma)}$ is equal to $\lambda \int_{0}^{t}\left(1+\gamma_{s}\right)\left(1-D_{s}\right) \mathrm{d} s$.

\subsection{Notation}

We now give a series of various notations which are used throughout the paper. In spite of being rather heavy, these notations are as simplified as possible and allow us to give explicit formulae for the different quantities characterizing the investment project.

(i) Let $\mathcal{N}$ be the cumulative distribution of the Gaussian law, i.e.

$$
\mathcal{N}(x)=\frac{1}{\sqrt{2 \pi}} \int_{-\infty}^{x} \mathrm{e}^{-t^{2} / 2} \mathrm{~d} t .
$$

(ii) For $s_{0}>0, v>0, L \geq 1, \varphi \geq 0$, and $a \in \mathbb{R}$, let $F_{\varphi}\left(s_{0}, v, L, a\right)$ be defined by

$$
\begin{aligned}
F_{\varphi}\left(s_{0}, v, L, a\right)= & \int_{0}^{+\infty} v \mathrm{e}^{-v t} \mathcal{N}\left(\frac{1}{\sigma \sqrt{t}} \ln \left(\frac{L}{s_{0}(1+\varphi)}\right)-a \sqrt{t}\right) \mathrm{d} t \\
& -\left(\frac{L}{s_{0}}\right)^{2 a / \sigma} \int_{0}^{+\infty} v \mathrm{e}^{-v t} \mathcal{N}\left(-\frac{1}{\sigma \sqrt{t}} \ln \left(\frac{L(1+\varphi)}{s_{0}}\right)-a \sqrt{t}\right) \mathrm{d} t
\end{aligned}
$$

and $\Delta F_{0, \varphi}\left(s_{0}, v, L, a\right)$ be defined by

$$
\Delta F_{0, \varphi}\left(s_{0}, v, L, a\right)=F_{\varphi}\left(s_{0}, v, L, a\right)-F_{0}\left(s_{0}, v, L, a\right) .
$$

(iii) For $v>0, \varphi \geq 0$, and $a \in \mathbb{R}$, let $f_{\varphi}(v, a)$ be defined by

$$
\begin{aligned}
f_{\varphi}(v, a)= & \int_{0}^{+\infty} v \mathrm{e}^{-v t} \frac{1}{\sqrt{2 \pi t}} \exp \left(-\frac{1}{2}\left(\frac{1}{\sigma \sqrt{t}} \ln (1+\varphi)+a \sqrt{t}\right)^{2}\right) \mathrm{d} t \\
& -a \int_{0}^{+\infty} v \mathrm{e}^{-v t} \mathcal{N}\left(-\frac{1}{\sigma \sqrt{t}} \ln (1+\varphi)-a \sqrt{t}\right) \mathrm{d} t
\end{aligned}
$$

and $\Delta f_{0, \varphi}(\nu, a)$ be defined by

$$
\Delta f_{0, \varphi}(v, a)=f_{\varphi}(v, a)-f_{0}(v, a) .
$$

(iv) We denote by $a_{0}, \theta_{0}$, and $v_{0}$ the following parameters

$$
\begin{aligned}
& a_{0}=\frac{1}{\sigma} \sqrt{\left(\alpha-\frac{\sigma^{2}}{2}\right)^{2}+2 \mu \sigma^{2}}, \\
& \theta_{0}=\frac{1}{\sigma}\left(\frac{\sigma^{2}}{2}-\alpha+\sqrt{\left.\left(\alpha-\frac{\sigma^{2}}{2}\right)^{2}+2 \mu \sigma^{2}\right)},\right. \\
& v_{0}=\frac{\alpha+\sigma^{2} / 2}{\sigma} .
\end{aligned}
$$




\section{Main results on optimal stopping and perpetual options}

This section is devoted to the study of the perpetual call option, described in Section 2.2. We first prove the existence of an optimal stopping time in (3), and characterize it as the hitting time of an optimal region. Then, we look at the different characteristics of the perpetual option. More precisely, we provide closed-form formulae for the Laplace transform of the optimal stopping time and for the real option value.

\subsection{Existence and characterization of an optimal stopping time}

In this section, we consider the question of the existence of an optimal stopping time for the problem (3). The following result holds true.

Theorem 1. There exists an optimal stopping time for the problem (3). In other words, there exists $\tau_{t}^{*} \in \Upsilon_{t}$ such that

$$
C(t, x, d)=\mathrm{E}\left(\mathrm{e}^{-\mu\left(\tau_{t}^{*}-t\right)}\left(S_{\tau_{t}^{*}}-1\right)^{+} \mid S_{t}=x, D_{t}=d\right) .
$$

Moreover, $\tau_{t}^{*}$ is characterized as the first hitting time by the process $S$ of a region $\left[L_{t}^{d, *},+\infty\right)$, fully known at time $t$, i.e.

$$
\tau_{t}^{*}=\inf \left\{s \geq t ; S_{s} \geq L_{t}^{d, *}\right\}
$$

Here, two very different situations have to be studied separately, depending on whether the jump has already occurred or not (in other words, whether $d=1$ or $d=0$ ) at time $t$ of the study. The arguments used in both cases are of a completely different nature.

3.1.1. Case 1: $D_{t}=1$. In the situation where the jump has occurred before time $t$, the problem

$$
C(t, x, 1)=\underset{\tau \in \Upsilon_{t}}{\operatorname{ess} \sup } \mathrm{E}\left(\mathrm{e}^{-\mu(\tau-t)}\left(S_{\tau}-1\right)^{+} \mid S_{t}=x, D_{t}=1\right)
$$

is standard and well known, therefore we simply state the results and sketch the proof. More precisely,

Proposition 1. (i) The optimal hitting time in the problem (9) exists and it is characterized as the first hitting time of a constant region $\left[L^{1, *},+\infty\right)$, i.e.

$$
\begin{aligned}
C(t, x, 1) & =\sup _{L \geq 1} \mathrm{E}\left(\mathrm{e}^{-\mu(\tau-t)}\left(S_{\tau_{L}}-1\right)^{+} \mid S_{t}=x, D_{t}=1\right) \\
& =\mathrm{E}\left(\mathrm{e}^{-\mu\left(\tau_{L^{1, *}}-t\right)}\left(S_{\tau_{L^{1, *}}}-1\right)^{+} \mid S_{t}=x, D_{t}=1\right),
\end{aligned}
$$

where $\tau_{L}=\inf \left\{t \geq 0 ; S_{t} \geq L\right\}$ is the first hitting time of $[L,+\infty)$.

(ii) The optimal level $L^{1, *}$ is given by

$$
L^{1, *}=\frac{k_{1}}{k_{1}-1}
$$

where $k_{1}$ is the unique positive real number such that $\psi\left(k_{1}\right)=\mu$, with $\psi$, the Lévy exponent of the Lévy process $X$, defined by

$$
X_{s}=\left(\alpha-\frac{1}{2} \sigma^{2}\right) s+\sigma W_{s} .
$$


Proof. Note that, on the set $\{t \geq T\}$, we necessarily have $\tau_{L} \geq T$ and, as a consequence, for any $s, t$ such that $0 \leq T \leq t \leq s$ we have

$$
S_{s}=x \exp \left(\left(\alpha-\frac{1}{2} \sigma^{2}\right)(s-t)+\sigma\left(W_{s}-W_{t}\right)\right) .
$$

Therefore, the proof of these results is similar to that of the standard Black-Scholes model (see, for example, Merton (1992, Section 8.8) or Karatzas and Shreve (1998, Section 2.6)), and we omit it.

3.1.2. Case 2: $D_{t}=0$. The second situation where the jump has not already occurred at time $t$ is not standard. The problem is of a double nature: first we have to prove the existence of an optimal stopping time for

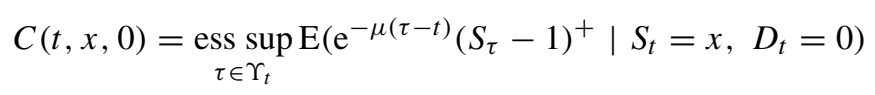

and then we have to characterize it.

(i) Existence of an optimal stopping time. For any $x \in \mathbb{R}_{*}^{+}$, and for $D_{t}=0$, we denote by $S^{t, x, 0}$ the trajectory of the process $S$ such that $S_{t}^{t, x, 0}=x$. Hence, for any $s \geq t$ we obtain

$$
S_{s}^{t, x, 0}=x(1+\Phi)^{D_{s}} \exp \left[\left(\alpha-\frac{1}{2} \sigma^{2}\right)(s-t)+\sigma\left(W_{s}-W_{t}\right)\right]
$$

The following result holds true.

Lemma 1. The stopping time

$$
\tau_{t}^{*}=\inf \left\{s \geq t \mid C\left(s, S_{s}^{t, x, 0}, 0\right)=\left(S_{s}^{t, x, 0}-1\right)^{+}\right\}
$$

is $\mathrm{P}$-almost surely finite.

Proof. Recall the constants $\underline{\Phi}$ and $\bar{\Phi}$ defined in Assumption 1. Now, the process $S \underline{\Phi}$ and the function $C \stackrel{\Phi}{ }$ are defined as follows:

$$
S_{s}^{\Phi}=x(1+\underline{\Phi}) \exp \left[\left(\alpha-\frac{1}{2} \sigma^{2}\right)(s-t)+\sigma\left(W_{s}-W_{t}\right)\right] \quad \text { for } s \geq t
$$

and

$$
C^{\Phi}(t, x)=\underset{\tau \in \Upsilon_{t}}{\operatorname{ess} \sup } \mathrm{E}\left(\mathrm{e}^{-\mu\left(\tau_{t}-t\right)}\left(S_{\tau_{t}}^{\Phi}-1\right)^{+} \mid S_{t}^{\Phi}=x(1+\underline{\Phi})\right) .
$$

The process $S^{\bar{\Phi}}$ and the function $C^{\bar{\Phi}}$ are defined in a similar way.

Because the inequalities $-1<\underline{\Phi} \leq \Phi<\bar{\Phi}$ hold true, we can write

$$
\begin{aligned}
\tau_{t}^{*} & \leq \inf \left\{s \geq t \mid C^{\bar{\Phi}}(t, x)=\left(S_{s}^{t, x, 0}-1\right)^{+}\right\} \\
& \leq \inf \left\{s \geq t \mid C^{\bar{\Phi}}(t, x)=\left(S_{s}^{\Phi}-1\right)^{+}\right\} \\
& \leq \inf \left\{s \geq t \mid C^{\bar{\Phi}}(t, x)=k\left(S_{s}^{\bar{\Phi}}-\frac{1}{k}\right)^{+}\right\},
\end{aligned}
$$

where $k=(1+\underline{\Phi}) /(1+\bar{\Phi})$. Now, from Assumption 1(iv) we have

$$
\inf \left\{s \geq t \mid C^{\bar{\Phi}}(t, x)=k\left(S_{s}^{\bar{\Phi}}-\frac{1}{k}\right)^{+}\right\}<\infty \quad \mathrm{P} \text { almost surely. }
$$


Proposition 2. There exists an optimal stopping time for the problem (10). In other words, there exists $\tau_{t}^{*} \in \Upsilon_{t}$ such that

$$
C(t, x, 0)=\mathrm{E}\left(\mathrm{e}^{-\mu\left(\tau_{t}^{*}-t\right)}\left(S_{\tau_{t}^{*}}-1\right)^{+} \mid S_{t}=x, D_{t}=0\right) .
$$

Proof. From (11) and Assumptions 1(i) and 1(iv), we deduce that the optimal stopping theory can apply (see, for example, El Karoui (1981, Section 2.11) or Peskir and Shiryayev (2006)); therefore we conclude from Lemma 1 that the stopping time $\tau_{t}^{*}$ is optimal.

(ii) Characterization of the optimal stopping time. In this second part of this case, we want to characterize the optimal stopping time of the problem (10). Using the same type of arguments as in Jacka (1991), we get a characterization similar to the one obtained for the case $D_{t}=1$.

Let $y$ be the continuation region defined by

$$
y=\left\{(t, x) \mid C(t, x, 0)>(x-1)^{+}\right\},
$$

and let $y_{t}$ denote the section

$$
y_{t}=\{x \mid(t, x) \in y\}
$$

We first give the characterization of the section $y_{t}$ of the continuation region in terms of an optimal frontier.

Proposition 3. For any $t>0$, there exists $L_{t}^{0, *}>1$ such that

$$
y_{t}=\left[0, L_{t}^{0, *}[\right.
$$

Proof. From (12), the function $x \mapsto C(t, x, 0)$ is continuous, therefore it suffices to prove the following implication:

$$
\left(x \in y_{t} \text { and } 0<y<x\right) \Longrightarrow\left(y \in y_{t}\right) .
$$

Let us assume that $x \in \mathcal{Y}_{t}$. As a consequence, $C(t, x, 0)>(x-1)^{+}$. Let $\tau_{t}^{x}$ be defined by

$$
\tau_{t}^{x}=\inf \left\{s \geq t \mid\left(s, S_{s}^{t, x, 0}\right) \notin \mathcal{Y}\right\} .
$$

On the one hand, we have,

$$
C(t, x, 0)=\mathrm{E}\left(\mathrm{e}^{-\mu\left(\tau_{t}^{x}-t\right)}\left(S_{\tau_{t}^{x}}-1\right)^{+} \mid S_{t}=x, D_{t}=0\right)=\mathrm{E}\left(\mathrm{e}^{-\mu\left(\tau_{t}^{x}-t\right)}\left(S_{\tau_{t}^{x}}^{t, x, 0}-1\right)^{+}\right)
$$

and, on the other hand, as $\tau_{t}^{x}$ is not optimal for $C(t, y, 0)$, we have

$$
C(t, y, 0) \geq \mathrm{E}\left(\mathrm{e}^{-\mu\left(\tau_{t}^{x}-t\right)}\left(S_{\tau_{t}^{x}}^{t, x, 0}-1\right)^{+}\right) .
$$

Therefore,

$$
C(t, x, 0)-C(t, y, 0) \leq \mathrm{E}\left(\mathrm{e}^{-\mu\left(\tau_{t}^{x}-t\right)}\left[\left(S_{\tau_{t}^{x}}^{t, x, 0}-1\right)^{+}-\left(S_{\tau_{t}^{x}}^{t, y, 0}-1\right)^{+}\right]\right) .
$$

The assumption $0<y<x$ together with (11) imply

$$
\begin{aligned}
& C(t, x, 0)-C(t, y, 0) \\
& \quad \leq \mathrm{E}\left(\mathrm{e}^{-\mu\left(\tau_{t}^{x}-t\right)}\left(S_{\tau_{t}^{x}}^{t, x, 0}-S_{\tau_{t}^{x}}^{t, y, 0}\right)\right) \\
& \quad \leq(x-y) \mathrm{E}\left(\exp \left((\alpha-\mu)\left(\tau_{t}^{x}-t\right)+\lambda \int_{t}^{\tau_{t}^{x}} \ln (1+\Phi)\left(1-D_{s}\right) \mathrm{d} s\right) \mathcal{E}(\sigma W)_{\tau_{t}^{y}, t}\right),
\end{aligned}
$$

where $\mathcal{E}(\sigma W)_{\tau_{t}^{y}, t}=\exp \left(\sigma\left(W_{\tau_{t}^{y}}-W_{t}\right)-\frac{1}{2} \sigma^{2}\left(\tau_{t}^{y}-t\right)\right)$. 
Let $\widetilde{\mathrm{P}}$ be the probability measure, equivalent to $\mathrm{P}$, defined by

$$
\frac{\mathrm{d} \widetilde{\mathrm{P}}}{\mathrm{dP}} \mid \mathcal{F}_{t}=\exp \left(\sigma W_{t}-\frac{1}{2} \sigma^{2} t\right)
$$

Now,

$C(t, x, 0)-C(t, y, 0) \leq(x-y) \widetilde{\mathrm{E}}\left(\exp \left((\alpha-\mu)\left(\tau_{t}^{x}-t\right)+\lambda \int_{t}^{\tau_{t}^{x}} \ln (1+\Phi)\left(1-D_{s}\right) \mathrm{d} s\right)\right)$.

From Assumption 1(iv), we have

$$
\widetilde{\mathrm{E}}\left(\exp \left((\alpha-\mu)\left(\tau_{t}^{x}-t\right)+\lambda \int_{t}^{\tau_{t}^{x}} \ln (1+\Phi)\left(1-D_{s}\right) \mathrm{d} s\right)\right) \leq 1
$$

and

$$
C(t, x, 0)-C(t, y, 0) \leq(x-y)
$$

or

$$
C(t, y, 0) \geq C(t, x, 0)-(x-y)>(x-1)^{+}-(x-y)>y-1 .
$$

Let $\xi=\inf \left\{u \geq t \mid S_{u}^{t, y, 0} \geq 2\right\}$, then

$$
\begin{aligned}
C(t, y, 0) & \geq \mathrm{E}\left(\mathrm{e}^{-\mu(\xi-t)}\left(S_{\xi}^{t, y, 0}-1\right)^{+}\right) \\
& \geq \mathrm{E}\left(\mathrm{e}^{-\mu(\xi-t)}\right) \\
& >0
\end{aligned}
$$

Therefore, for any $(t, y) \in \mathbb{R}^{+} \times \mathbb{R}_{*}^{+}, C(t, y, 0)>0$. Finally, $C(t, y, 0)>(y-1)^{+}$. Hence, the result follows.

We can now characterize the optimal stopping time as the hitting time of a region.

Corollary 1. The optimal stopping time $\tau_{t}^{*}$ of the problem (10) is characterized by

$$
\tau_{t}^{*}=\inf \left\{s \geq t \mid S_{s}^{t, x, 0} \geq L_{t}^{0, *}\right\}
$$

where $L_{t}^{0, *}$ is defined in Proposition 3.

Proof. The proof is an immediate consequence of Proposition 3.

\subsection{Perpetual option characteristics}

We are now interested in finding the perpetual option characteristics, in particular its value. As previously, the situations when $D_{t}=1$ and $D_{t}=0$ are very different. Therefore, we will look at them separately.

3.2.1. Case 1: $D_{t}=1$. As previously mentioned, when the jump has already occurred before time $t$, the results are standard in this case.

Proposition 4. (i) The Laplace transform of the optimal time is

$$
\mathrm{E}\left(\mathrm{e}^{-\mu\left(\tau_{L^{1, *}}-t\right)} \mid S_{t}=x, D_{t}=1\right)=\left(\frac{x}{L^{1, *}}\right)^{L^{1, *} / L^{1, *}-1},
$$


(ii) The price of the real option is given by

$$
C(t, x, 1)=\left(L^{1, *}-1\right)\left(\frac{L^{1, *}}{x}\right)^{-k_{1}},
$$

where the optimal level $L^{1, *}$ is given by Proposition 1.

Proof. These results are similar to those of the standard Black-Scholes framework and can be found, for example, in Merton (1992) or Karatzas and Shreve (1998).

Note that the formulae do not apparently depend on the jump size $\Phi$. This comes from the fact that after the jump, the jump size does not play any role apart from the shift in the 'initial condition', which becomes $s_{0}(1+\Phi)$ instead of $s_{0}$. This dependency is somehow hidden in the value $x$ of the process at time $t$.

3.2.2. Case 2: $D_{t}=0$. As previously, the situation where the jump has not occurred yet at time $t$, is not standard. We now want to study the characteristics of the perpetual option

$$
C(t, x, 0)=\underset{\tau \in \Upsilon_{t}}{\operatorname{ess} \sup } \mathrm{E}\left(\mathrm{e}^{-\mu(\tau-t)}\left(S_{\tau}-1\right)^{+} \mid S_{t}=x, D_{t}=0\right),
$$

which can also be written, using Corollary 1 , as

$$
C(t, x, 0)=\underset{L_{t}^{0} \geq 1}{\operatorname{ess} \sup } \mathrm{E}\left(\mathrm{e}^{-\mu\left(\tau L_{t}^{0,-t)}\right.}\left(S_{\tau_{L_{t}^{0}}}-1\right)^{+} \mid S_{t}=x, D_{t}=0\right) .
$$

For the sake of simplicity in the notation and computation but without loss of generality, we consider this problem at time $t=0$ (obviously, $D_{0}=0$ ). Therefore, we look at

$$
C\left(0, s_{0}, 0\right)=\underset{L^{0} \geq 1}{\operatorname{ess} \sup } \mathrm{E}\left(\mathrm{e}^{-\mu \tau_{L^{0}}}\left(S_{\tau_{L^{0}}}-1\right)^{+} \mid S_{0}=s_{0}\right) .
$$

We now present the main result of this paper, giving the perpetual option characteristics in a general framework where we do not know a priori the sign of the jump size $\Phi$. The following two theorems provide these explicit formulae at time zero. In particular, the optimal frontier and a characterization of the optimal hitting time are now given in Theorem 2.

Theorem 2. (i) The optimal frontier of the problem (13) is given by

$$
L_{\Phi}^{0, *}=\frac{\eta(\Phi)}{\eta(\Phi)-1+\Gamma(\Phi)}
$$

where

$$
\begin{aligned}
\eta(\Phi)= & \frac{2}{\sigma} f_{0}\left(\lambda, a_{0}\right)+\theta_{0} \sigma \\
& -\frac{2}{\sigma} \mathrm{E}\left(\mathbf{1}_{\{\Phi<0\}}(1+\Phi)^{\theta_{0} / \sigma}\right) f_{0}\left(\lambda, a_{0}\right) \\
& +\frac{2}{\sigma} \mathrm{E}\left[\mathbf{1}_{\{\Phi>0\}}\left[\frac{\lambda}{\lambda+\mu} \Delta f_{0, \Phi}\left(\lambda+\mu, a_{0}-\theta_{0}\right)-(1+\Phi)^{\theta_{0} / \sigma} f_{\Phi}\left(\lambda, a_{0}\right)\right]\right]
\end{aligned}
$$

and

$\Gamma(\Phi)$

$$
=\frac{2}{\sigma} \mathrm{E}\left[\mathbf{1}_{\{\Phi>0\}}\left(\frac{\lambda(1+\Phi)}{\lambda+\mu-\alpha} \Delta f_{0, \Phi}\left(\lambda+\mu-\alpha, v_{0}\right)-\frac{\lambda}{\lambda+\mu} \Delta f_{0, \Phi}\left(\lambda+\mu, a_{0}-\theta_{0}\right)\right)\right] .
$$


(ii) For every $s_{0}$ such that $0<s_{0}<L_{\Phi}^{0, *}$, the optimal hitting time is characterized by its Laplace transform

$$
\mathrm{E}\left(\exp \left(-\mu \tau_{L_{0}^{0, *}(\Phi)}\right)\right)=\left(\frac{s_{0}}{L_{\Phi}^{0, *}}\right)^{\theta_{0} / \sigma}(1+K(\Phi))+\Theta(\Phi)
$$

where

$$
\begin{aligned}
K(\Phi)= & -F_{0}\left(s_{0}, \lambda, L_{\Phi}^{0, *}, a_{0}\right)+F_{0}\left(s_{0}, \lambda, L_{\Phi}^{0, *}, a_{0}\right) \mathrm{E}\left[\mathbf{1}_{\{\Phi<0\}}(1+\Phi)^{\theta_{0} / \sigma}\right] \\
& +\mathrm{E}\left[\mathbf{1}_{\{\Phi>0\}}(1+\Phi)^{\theta_{0} / \sigma} F_{\Phi}\left(s_{0}, \lambda, L_{\Phi}^{0, *}, a_{0}\right)\right], \\
\Theta(\Phi)= & -\frac{\lambda}{\lambda+\mu} \mathrm{E}\left[\mathbf{1}_{\{\Phi>0\}} \Delta F_{0, \Phi}\left(s_{0}, \lambda+\mu, L_{\Phi}^{0, *}, a_{0}-\theta_{0}\right)\right] ;
\end{aligned}
$$

see Section 2.3 for the notation.

Proof. Let $\Phi$ be a constant denoted by $\varphi$. To prove this result, we first consider the following two cases: $\varphi$ is positive (i.e. a bullish environment) or $\varphi$ is negative (i.e. a bearish environment). Finally, the general result is obtained by means of conditional expectation, as it is enough to consider the case where the jump size is a constant, from the independence of the random variable $\Phi$ and the filtration generated by the Brownian motion and the jump process.

(a) The bullish environment. Assuming the random variable $\Phi$ to be a positive constant $\varphi$, we determine the optimal threshold on which the agent bases his decision. We proceed in two steps to obtain $L_{\Phi}^{0, *}$ in a bullish framework (with $\varphi>0$ ).

Step 1. The functions $F_{\varphi}$ and $f_{\varphi}$, defined by (4) and (5) are linked by the following relationship:

$$
\text { for all } \lambda>0 \text { and for all } a \in \mathbb{R},\left.\quad \frac{\partial}{\partial L} F_{\varphi}\left(s_{0}, \lambda, L, a\right)\right|_{s_{0}}=L=\frac{2}{\sigma L} f_{\varphi}(\lambda, a) .
$$

Moreover, the function $F_{\varphi}$ satisfies $F_{\varphi}(L, \lambda, L, a)=0$.

Step 2. We denote by $c$ the following function:

$$
c\left(s_{0}, L\right)=\mathrm{E}\left[\mathrm{e}^{-\mu \tau_{L}}\left(S_{\tau_{L}}-1\right)\right] .
$$

Then from Lemma 2 we deduce the following:

$$
\begin{aligned}
\left.\frac{\partial}{\partial L} c\left(s_{0}, L\right)\right|_{s_{0}}=L \\
=\frac{2}{\sigma L}(1+\varphi)^{\theta_{0} / \sigma}(L-1) f_{\varphi}\left(\lambda, a_{0}\right)+1-\frac{\theta_{0}}{\sigma L}(L-1)-\frac{2}{\sigma L}(L-1) f_{0}\left(\lambda, a_{0}\right) \\
\quad-\frac{2 \lambda(1+\varphi)}{\sigma(\lambda+\mu-\alpha)} \Delta f_{0, \varphi}\left(\lambda+\mu-\alpha, v_{0}\right)+\frac{2 \lambda}{\sigma L(\lambda+\mu)} \Delta f_{0, \varphi}\left(\lambda+\mu, a_{0}-\theta_{0}\right) .
\end{aligned}
$$

The unique solution of

$$
\left.\frac{\partial}{\partial L} c\left(s_{0}, L\right)\right|_{s_{0}}=L=0
$$

is

$$
L=L_{\varphi}^{0, *}=\frac{\eta^{+}(\varphi)}{\eta^{+}(\varphi)-1+\Gamma^{+}(\varphi)}
$$


where

$$
\begin{aligned}
\eta^{+}(\varphi) & =\frac{2}{\sigma} f_{0}\left(\lambda, a_{0}\right)+\theta_{0} \sigma+\frac{2}{\sigma}\left[\frac{\lambda}{\lambda+\mu} \Delta f_{0, \varphi}\left(\lambda+\mu, a_{0}-\theta_{0}\right)-(1+\varphi)^{\theta_{0} / \sigma} f_{\varphi}\left(\lambda, a_{0}\right)\right], \\
\Gamma^{+}(\varphi) & =\frac{2}{\sigma}\left[\frac{\lambda(1+\Phi)}{\lambda+\mu-\alpha} \Delta f_{0, \varphi}\left(\lambda+\mu-\alpha, v_{0}\right)-\frac{\lambda}{\lambda+\mu} \Delta f_{0, \varphi}\left(\lambda+\mu, a_{0}-\theta_{0}\right)\right] .
\end{aligned}
$$

(b) The bearish environment. In the case where the constant $\varphi$ is nonpositive, the optimal frontier is given by

$$
L_{\varphi}^{0, *}=\frac{\eta^{-}(\varphi)}{\eta^{-}(\varphi)-1},
$$

where

$$
\eta^{-}(\varphi)=\frac{2}{\sigma}\left[\left(1-(1+\varphi)^{\theta_{0} / \sigma}\right) f_{0}\left(\lambda, a_{0}\right)+\frac{\theta_{0}}{2}\right] .
$$

The proof for this case is similar to that of the previous case and the result follows directly from Lemma 3.

Theorem 2(i) is then a straightforward consequence of parts (a) and (b) of the proof and of the independence of the random variable $\Phi$ with respect to the Brownian motion and the jump process. Theorem 2(ii) can be deduced from this independence, Lemma 2, Lemma 3, and the value of the optimal threshold given in Theorem 2(i).

The following theorem gives the perpetual option value.

Theorem 3. For every $s_{0}$ such that $0<s_{0}<L_{\Phi}^{0, *}$, the perpetual option value at time $t=0$ is

$$
C\left(0, s_{0}, 0\right)=\left(L_{\Phi}^{0, *}-1\right)\left(\frac{s_{0}}{L_{\Phi}^{0, *}}\right)^{\theta_{0} / \sigma}(1+K(\Phi))+J(\Phi),
$$

where $K(\cdot)$ is defined in Theorem 2 and

$$
J(\Phi)=-\frac{\lambda s_{0}}{\lambda+\mu-\alpha} \mathrm{E}\left[\mathbf{1}_{\{\Phi>0\}}(1+\Phi) \Delta F_{0, \Phi}\left(s_{0}, \lambda+\mu-\alpha, L_{\Phi}^{0, *}, v_{0}\right)\right] ;
$$

see Section 2.3 for the notation.

Proof. The proof follows directly from Theorem 2, Lemma 2, and Lemma 3.

\subsection{Comments}

The model presented here, in particular the situation $D_{t}=0$, is very different from the existing literature, as it corresponds to a non-Lévy model. The results are therefore different. Note, however, that if $\Phi \equiv 0 \mathrm{P}$ almost surely, then all the formulae of this study are similar to those of the 'Black-Scholes' model. The 'interesting' situation is when $D_{t}$ is different from 1. Otherwise, the formulae are similar to a standard Black-Scholes-type framework, where no possible jump of the underlying process is introduced (as it is equivalent to have a shift in the initial condition of our problem). Therefore, we focus on the nonstandard results, when $D_{t}=0$.

In this paper, Theorem 2 gives an explicit representation of the optimal boundary without any particular assumption either on the sign of the jump $\Phi$ or on its distribution. Whether $\Phi$ is positive or negative has a great impact on the structure of the different results since it shapes the optimal frontier differently. In fact, both situations are mathematically very different, as it can 
be seen when considering the two particular cases where the random variable $\Phi$ has a constant negative versus positive sign. The main reason is that when the jump is negative, the process $S$ necessarily crosses the given frontier in a continuous way. However, this property does not hold any more if the jump is positive. Therefore, the mathematics involved and the results we obtain are dissimilar. Moreover, the results we obtain are strongly different from those of the Lévy model.

When the jump is negative, even if the optimal frontier is still of a standard shape, i.e.

$$
L_{\Phi}^{0, *}=\frac{\eta^{-}(\Phi)}{\eta^{-}(\Phi)-1},
$$

both the Laplace transform and the value of the investment opportunity are different. The standard equations for the Laplace transform and the price are perturbed by a multiplicative factor $K$, this factor $K$ being identical to the one in the bullish environment, we have

$$
\begin{aligned}
& \mathrm{E}\left(\exp \left(-\mu \tau_{L_{0}^{0, *}(\Phi)}\right)\right)=\left(\frac{s_{0}}{L_{\Phi}^{0, *}}\right)^{L_{\Phi}^{0, *} /\left(L_{\Phi}^{0, *}-1\right)}(1+K(\Phi)), \\
& C\left(0, s_{0}, 0\right)=\left(L_{\Phi}^{0, *}-1\right)\left(\frac{s_{0}}{L_{\Phi}^{0, *}}\right)^{L_{\Phi}^{0, *} /\left(L_{\Phi}^{0, *}-1\right)}(1+K(\Phi)) .
\end{aligned}
$$

This multiplicative factor comes from the memory of the process used to model the profit and cost ratio. Obviously, in the limit situation where there is no possible jump $(\Phi \equiv 0)$, the results coincide with those of the standard Black-Scholes-type framework as $K(0)=0$.

When considering a positive jump, we obtain explicit formulae for the three characteristics of the investment opportunity. The results we obtain are fundamentally different from those of Lévy models. In particular, the optimal frontier cannot be written under the previous standard form (if we exclude the particular limit case where $\Phi=0$ ). Indeed, an additional term $\Gamma^{+}(\Phi)$ is present in the denominator of the optimal frontier, i.e.

$$
L_{\Phi}^{0, *}=\frac{\eta^{+}(\Phi)}{\eta^{+}(\Phi)-1+\Gamma^{+}(\Phi)} .
$$

Both the Laplace transform and the investment value differ from the standard case by two different effects. There is first a common multiplicative factor $K$ and also two additive factors $J^{\prime}$ (for the Laplace transform) and $J$ (for the value of the investment), i.e.

$$
\begin{aligned}
& \mathrm{E}\left(\exp \left(-\mu \tau_{L_{0}^{0, *}(\Phi)}\right)\right)=\Theta(\Phi)+\left(\frac{s_{0}}{L_{\Phi}^{0, *}}\right)^{L_{\Phi}^{0, *} /\left(L_{\Phi}^{0, *}-1\right)}(1+K(\Phi)) \\
& C\left(0, s_{0}, 0\right)=J(\Phi)+\left(L_{\Phi}^{0, *}-1\right)\left(\frac{s_{0}}{L_{\Phi}^{0, *}}\right)^{L_{\Phi}^{0, *} /\left(L_{\Phi}^{0, *}-1\right)}(1+K(\Phi))
\end{aligned}
$$

These differences can be explained as follows. As for the bearish case, the factor $K$ comes from the memory of the process used to model the profit and cost ratio, whereas the additive factors $J^{\prime}$ and $J$ translate the fact that in this case the optimal boundary $L_{\Phi}^{0, *}$ is not necessarily crossed in a continuous way. 


\section{Application to a real option problem}

In this section, we look more closely at the investment problem (i.e. the value of the investment project and optimal hitting time) when the investor has no particular knowledge about the type of impact a new regulation, i.e. a sudden shock, may have on the project itself. Namely, we do not know whether the sign of the jump will be positive or negative. This may be the case for investment projects related to new technologies when the prospects are uncertain. In this case, the related market witnesses a rapid evolution. New regulations can be adopted, modifying its structure. They can generate either a positive impact when improving the security and the transparency (bullish environment) or a negative impact when increasing its complexity (bearish environment). New competitors can also enter the market, attracted by these new prospects, and actual competitors can default, victims of a lack of experience and speculation. Finally, we may think of new techniques that can be patented either by the considered firm or by competitors.

In this section, we focus on the situation at time $t=0$ without any loss of generality. In fact, if a new regulation is expected, or if some disruptive factor for the economic environment is likely to occur, we may think that the investor will delay his investment decision and wait for the actual occurrence of the shock to evaluate the impact on the investment project and to finally take his decision. This delay seems to be all the more important since the potential impact (positive or negative) is unknown. In reality, however, the investor does not necessarily have the opportunity of delaying his decisions, and often has to decide as soon as the investment project appears (i.e. at time $t=0$ ). This pressure may be due to several reasons. In the first place, the investor could face some legal constraints, for instance in the search for concessions or licences (for example, the Universal Mobile Telecommunications Systems, where the investors have to take their decisions before a fixed deadline). The investor can also face some market and competition constraints, where the postponement of a decision can give his competitors the opportunity of taking a stand and, as a consequence, invalidate any possibility of investing in the strategic business field for the investor. So considering the study at time $t=0$ appears to be relevant for the investment problem we analyze.

In the following, we look at a real option problem in a disrupted framework, as previously described. We illustrate the results of Section 3 and provide numerical applications. In such a context, one of the most important questions is certainly related to the calibration of the model. This is all the more important since the shock induces a drastic decrease in the project cash flows. Therefore, we conclude this study by looking at the impact of a model misspecification in a bearish environment, i.e. when the impact of a shock is to be negative.

\subsection{Illustration}

We now illustrate the main results of this paper (Theorems 2 and 3) through numerical examples. More precisely, we consider an agent facing an investment problem at time zero, in a potentially disturbed environment. We focus especially on the sensitivity with respect to the jump size of both the Laplace transform of the hitting time and the investment value. The set of parameters we consider is

$$
s_{0}=0.8, \quad \sigma=0.2, \quad \alpha=0.05, \quad \mu=0.15 .
$$

In Table 1 we give some values of the optimal profit and cost ratio for different values of the jump intensity $\lambda$ and the jump size $\varphi$. The optimal ratio is monotonic with respect to the jump size $\varphi$. Moreover, the optimal ratio is a nonincreasing function of the jump intensity $\lambda$ for negative values of $\varphi$, whereas it is a nondecreasing function of $\lambda$ for positive values of the 
TABLE 1: The optimal profit-cost ratio.

\begin{tabular}{lllll}
\hline \multicolumn{1}{c}{$\varphi$} & $\lambda=0.25$ & $\lambda=0.5$ & $\lambda=1$ & $\lambda=2$ \\
\hline-0.99 & 1.407617 & 1.37467 & 1.364417 & 1.367871 \\
-0.8 & 1.415605 & 1.382496 & 1.372175 & 1.375653 \\
-0.6 & 1.443986 & 1.410464 & 1.399953 & 1.403498 \\
-0.5 & 1.468846 & 1.435171 & 1.424556 & 1.428139 \\
-0.4 & 1.503957 & 1.470402 & 1.459746 & 1.463347 \\
-0.3 & 1.553697 & 1.520998 & 1.510504 & 1.514057 \\
-0.2 & 1.625893 & 1.595907 & 1.586132 & 1.58945 \\
-0.1 & 1.735855 & 1.713473 & 1.705999 & 1.708546 \\
-0.05 & 1.814285 & 1.800001 & 1.795146 & 1.796805 \\
0 & 1.917891 & 1.917891 & 1.917891 & 1.917891 \\
0.05 & 2.061146 & 2.098451 & 2.13082 & 2.165905 \\
0.1 & 2.259259 & 2.38787 & 2.545456 & \\
0.15 & 2.530455 & 2.851521 & & \\
0.2 & 2.90661 & 3.639258 & & \\
0.25 & 3.446203 & & & \\
0.3 & 4.267252 & & & \\
0.35 & 5.645627 & & & \\
0.4 & 8.406155 & & & \\
\hline
\end{tabular}

TABLE 2: The Laplace transform of the optimal time to invest.

\begin{tabular}{lllll}
\hline \multicolumn{1}{c}{$\varphi$} & $\lambda=0.25$ & $\lambda=0.5$ & \multicolumn{1}{c}{$\lambda=1$} & \multicolumn{1}{c}{$\lambda=2$} \\
\hline-0.99 & 0.117852 & 0.066776 & 0.025591 & 0.00582 \\
-0.9 & 0.118797 & 0.068404 & 0.027797 & 0.008327 \\
-0.8 & 0.121859 & 0.073688 & 0.034962 & 0.016465 \\
-0.7 & 0.127039 & 0.08268 & 0.047158 & 0.030297 \\
-0.6 & 0.13414 & 0.09513 & 0.064061 & 0.049423 \\
-0.5 & 0.142747 & 0.11047 & 0.084925 & 0.07296 \\
-0.4 & 0.152147 & 0.127691 & 0.10844 & 0.099398 \\
-0.3 & 0.161184 & 0.145118 & 0.132457 & 0.126331 \\
-0.2 & 0.167994 & 0.159997 & 0.153506 & 0.149993 \\
-0.1 & 0.169553 & 0.167775 & 0.165981 & 0.164525 \\
-0.05 & 0.166918 & 0.166801 & 0.166368 & 0.165701 \\
0 & 0.160903 & 0.160903 & 0.160903 & 0.160903 \\
0.05 & 0.150273 & 0.146763 & 0.142857 & 0.138179 \\
0.1 & 0.135036 & 0.123248 & 0.10860 & \\
0.15 & 0.116245 & 0.093441 & & \\
0.2 & 0.095067 & 0.061498 & & \\
0.25 & 0.072806 & & & \\
0.3 & 0.050901 & & & \\
0.35 & 0.030943 & & & \\
0.4 & 0.014657 & & &
\end{tabular}


TABLE 3: The investment value.

\begin{tabular}{llccc}
\hline \multicolumn{1}{c}{$\lambda$} & $\lambda=0.25$ & $\lambda=0.5$ & $\lambda=1$ & $\lambda=2$ \\
\hline-0.99 & 0.048038 & 0.025019 & 0.009326 & 0.002141 \\
-0.9 & 0.048642 & 0.025752 & 0.010179 & 0.003078 \\
-0.8 & 0.050645 & 0.028185 & 0.013012 & 0.006185 \\
-0.7 & 0.054217 & 0.032532 & 0.018065 & 0.011712 \\
-0.6 & 0.059556 & 0.039048 & 0.025622 & 0.019942 \\
-0.5 & 0.066926 & 0.048073 & 0.036055 & 0.031237 \\
-0.4 & 0.076676 & 0.060066 & 0.049855 & 0.046056 \\
-0.3 & 0.089247 & 0.075606 & 0.067620 & 0.064941 \\
-0.2 & 0.105146 & 0.095343 & 0.089975 & 0.088414 \\
-0.1 & 0.124766 & 0.119703 & 0.117183 & 0.116573 \\
-0.05 & 0.135919 & 0.133441 & 0.132287 & 0.132032 \\
0 & 0.147691 & 0.147691 & 0.147691 & 0.147691 \\
0.05 & 0.159882 & 0.161456 & 0.161618 & 0.161111 \\
0.1 & 0.171207 & 0.171558 & 0.167923 & \\
0.15 & 0.179599 & 0.173483 & & \\
0.2 & 0.183058 & 0.16257 & & \\
0.25 & 0.179627 & & & \\
0.3 & 0.167352 & & & \\
0.35 & 0.144303 & & & \\
0.4 & 0.10875 & & &
\end{tabular}

jump size. Note also that whatever the jump intensity is, this ratio tends to

$$
\frac{\theta_{0}}{\left(\theta_{0}-\sigma\right)}
$$

when the jump size tends to zero. This value corresponds to the optimal ratio when the model uncertainty is simply driven by a Brownian motion.

Table 2 provides the variation of the Laplace transform $\mathrm{E}\left(\exp \left(-\mu \tau_{L_{\varphi}^{0, *}}\right)\right)$ and those of the optimal investment value at time zero with respect to the jump size $\varphi$ for different values of $\lambda$.

Tables 2 and 3 provide highly dissymmetrical values with respect to the sign of the jump. Neither the Laplace transform nor the value of the investment are monotonic with respect to $\varphi$. If the jump size tends to zero, then the Laplace transform tends to $\left(s_{0}\left(\theta_{0}-\sigma\right) / \theta_{0}\right)^{\theta_{0} / \sigma}$, and it coincides with the value of the Laplace transform of the optimal hitting time in a Brownian model. The same result holds for the investment value.

\subsection{Misspecification impact in a bearish environment}

Assume now that the agent who does not know the (negative) random jump size $\Phi$, can however estimate it by its expectation $\mathrm{E}(\Phi)$. The question is then if he takes his investment decision by considering only the expected value, how will his decision be impacted, in particular his investment timing: will he invest too early or too late in the project? Note that the value of the investment project is obviously reduced since $C\left(0, s_{0}, 0\right)$ is determined by using an optimal frontier satisfying the supremum, i.e.

$$
C\left(0, s_{0}, 0\right)=\underset{L^{0} \geq 1}{\operatorname{ess} \sup } \mathrm{E}\left(\mathrm{e}^{-\mu \tau_{L^{0}}}\left(S_{\tau_{L^{0}}}-1\right)^{+} \mid S_{0}=s_{0}\right) .
$$


Any other frontier (in particular the level obtained by considering the expected value of the jump size instead of the random variable) is suboptimal, and the supremum is not reached for this particular value.

Let us first focus on the optimal level of benefit and cost ratio when the agent considers the expected value as the estimate for the jump size. In the following result we establish that such a misspecification leads the investor to undervalue the optimal level of the benefit/cost ratio.

Proposition 5. Using previous notation, we have

$$
L_{\Phi}^{0, *} \geq L_{\mathrm{E}(\Phi)}^{0, *}
$$

Proof. Let $g$ be the function defined by

$$
g(\theta)=\frac{1}{2} \theta^{2}+\frac{\theta}{\sigma}\left(\alpha-\frac{\sigma^{2}}{2}\right)-\mu .
$$

The inequalities $0<\theta_{0}$ and $0 \leq \alpha<\mu$ and the equalities $g\left(\theta_{0}\right)=0$ and $g(\sigma)=\alpha-\mu$ imply that $\theta_{0} / \sigma>1$. Hence, by Jensen's inequality, we deduce

$$
\mathrm{E}\left((1+\Phi)^{\theta_{0} / \sigma}\right) \geq(1+\mathrm{E}(\Phi))^{\theta_{0} / \sigma} .
$$

On the other hand, we have

$$
f_{0}(\lambda, a) \geq 0 \quad \text { for all } \lambda>0 \text { and for all } a \in \mathbb{R} .
$$

So, from Theorem 2, we may conclude that

$$
L_{\Phi}^{0, *} \geq L_{\mathrm{E}(\Phi)}^{0, *}
$$

Therefore, assuming that the agent chooses his investment time according to $L_{\mathrm{E}(\Phi)}^{0, *}$, he will enter the project as soon as the benefit/cost ratio reaches this threshold (instead of using the optimal threshold $\left.L_{\Phi}^{0, *}\right)$. In that sense, it can be said that the model misspecification leads them to undertake the project too early. More precisely, the agent has some beliefs regarding the dynamics of the random benefit/cost ratio. He knows perfectly its dynamics when his information is perfect or has simply an estimation when his access to the information is limited. Given his beliefs, the agent is able to explicitly determine the optimal ratio and therefore his investment strategy using this ratio as the threshold to take his decision. He has no better way to decide when it is optimal to invest, as the knowledge of the dynamics of $S$ simply gives him the Laplace transform of the optimal investment time and therefore simply a heuristic determination of an average investment time (see, for example, Barrieu and Bellamy (2005)).

\section{Conclusion}

In this paper, We provide closed-form formulae for the different characteristics of a perpetual option in a framework where the uncertainty is brought by a Brownian motion and a point process with stochastic intensity. An application of these results to a real option problem is then presented. Assuming that a unique shock occurs in the context of an investment project, we study the investment problem when the project's environment can be subject to a brutal and fundamental change (for instance, in its regulation or in its competitors' strengths and numbers). Mathematically speaking, all these situations are similar and are equivalent to 
considering a single jump diffusion process. Our aim has been to study the impact of such a market perturbation on the decision. More precisely, assuming that the investor has a budget allocation problem at time zero, he has to determine whether the project is interesting and if so, to have some idea of the optimal investment time as to allocate his capital among financial instruments with different maturities. In this framework, the investor determines his strategy at time zero by characterizing the optimal profit/cost ratio, and then can anticipate the optimal time to invest in the project by looking at the Laplace transform at time zero of the first hitting time of this optimal ratio. We have obtained closed-form formulae for the optimal benefit/cost ratio, the investment value, and the optimal time to enter the project, without any assumption on the distribution of the jump size and with arbitrary positive or negative jump.

The framework of the investment problem studied in this paper can be extended. For example, it is also possible to consider an investment environment that could be altered potentially by different shocks. In this case, the profit/cost ratio is then written in the form

$$
\mathrm{d} S_{t}=S_{t^{-}}\left(\alpha \mathrm{d} t+\sigma \mathrm{d} W_{t}+\sum_{i=1}^{i=k} \Phi_{i} \mathrm{~d} D_{i t}\right)
$$

where $D_{i t}=1_{t \geq T_{i}}$ and $T_{1}, T_{2}-T_{3}, \ldots, T_{k}-T_{k-1}$ is an increasing sequence of independent random variables with exponential law, the parameter of $T_{i}-T_{i-1}$ being $\lambda_{i}$. Such an extension seems rather natural as it leads to a framework allowing for different impacts of different natures on the investment field. In this sense, the uncertainty prevailing in investment problems could be represented.

\section{Appendix A}

Lemma 2. Assuming that the jump size is a positive constant $\varphi$, the following equalities hold:

$$
\begin{aligned}
& \mathrm{E}\left(\mathrm{e}^{-\mu \tau_{L}} \mathbf{1}_{\left\{\tau_{L}=T\right\}}\right)=-\frac{\lambda}{\lambda+\mu} \Delta F_{0, \varphi}\left(s_{0}, \lambda+\mu, L, a_{0}-\theta_{0}\right), \\
& \mathrm{E}\left(\mathrm{e}^{-\mu \tau_{L}} \mathbf{1}_{\left\{\tau_{L}>T\right\}}\right)=\left(\frac{(1+\varphi) s_{0}}{L}\right)^{\theta_{0} / \sigma} F_{\varphi}\left(s_{0}, \lambda, L, a_{0}\right), \\
& \mathrm{E}\left(\mathrm{e}^{-\mu \tau_{L}} \mathbf{1}_{\left\{\tau_{L}<T\right\}}\right)=\left(\frac{s_{0}}{L}\right)^{\theta_{0} / \sigma}\left[1-F_{0}\left(s_{0}, \lambda, L, a_{0}\right)\right], \\
& c\left(s_{0}, L\right)= \mathrm{E}\left[\mathrm{e}^{-\mu \tau_{L}}\left(S_{\tau}-1\right)\right] \\
&=(L-1)\left(\frac{s_{0}}{L}\right)^{\theta_{0} / \sigma}\left[1-F_{0}\left(s_{0}, \lambda, L, a_{0}\right)\right] \\
&+(L-1)\left(\frac{(1+\varphi) s_{0}}{L}\right)^{\theta_{0} / \sigma} F_{\varphi}\left(s_{0}, \lambda, L, a_{0}\right) \\
&-\frac{\lambda s_{0}(1+\varphi)}{\lambda+\mu-\alpha} \Delta F_{0, \varphi}\left(s_{0}, \lambda+\mu-\alpha, L, v_{0}\right) \\
&+\frac{\lambda}{\lambda+\mu} \Delta F_{0, \varphi}\left(s_{0}, \lambda+\mu, L, a_{0}-\theta_{0}\right) .
\end{aligned}
$$


Proof. (i) We have

$$
\mathrm{E}\left(\mathrm{e}^{-\mu \tau_{L}} \mathbf{1}_{\left\{\tau_{L}=T\right\}}\right)=\frac{\lambda}{\lambda+\mu} \mathrm{E}^{\psi_{1}}\left(\mathbf{1}_{\left\{\tau_{L}=T\right\}}\right)
$$

where $\psi_{1}=\mu / \lambda$ and where $\mathrm{P}^{\psi_{1}}$ is the P-equivalent probability measure specified by its Radon-Nikodym derivative

$$
\left.\frac{\mathrm{dP}^{\psi_{1}}}{\mathrm{dP}}\right|_{\mathcal{F}_{t}}=\mathcal{E}\left(\psi_{1} M\right)_{t}=\exp \left(\ln \left(1+\psi_{1}\right) D_{t}-\lambda \psi_{1} \int_{0}^{t}\left(1-D_{s}\right) \mathrm{d} s\right),
$$

and $\mathrm{E}^{\psi_{1}}$ is the $\mathrm{P}^{\psi_{1}}$-expectation. Hence, we obtain

$$
\begin{aligned}
\mathrm{E}^{\psi_{1}}\left(\mathbf{1}_{\left\{\tau_{L}=T\right\}}\right)= & \int_{0}^{+\infty}(\lambda+\mu) \mathrm{e}^{-(\lambda+\mu) t}\left[\mathcal{N}\left(\frac{y-u_{0} t}{\sqrt{t}}\right)-\mathrm{e}^{2 u_{0} y} \mathcal{N}\left(\frac{-y-u_{0} t}{\sqrt{t}}\right)\right] \mathrm{d} t \\
& -\int_{0}^{+\infty}(\lambda+\mu) \mathrm{e}^{-(\lambda+\mu) t}\left[\mathcal{N}\left(\frac{x-u_{0} t}{\sqrt{t}}\right)-\mathrm{e}^{2 u_{0} y} \mathcal{N}\left(\frac{x-2 y-u_{0} t}{\sqrt{t}}\right)\right] \mathrm{d} t
\end{aligned}
$$

we finally obtain

$$
\begin{aligned}
\mathrm{E}\left(\mathrm{e}^{-\mu \tau_{L}} \mathbf{1}_{\left\{\tau_{L}=T\right\}}\right) & =\frac{\lambda}{\lambda+\mu}\left(F_{0}\left(s_{0}, \lambda+\mu, L, u_{0}\right)-F_{\varphi}\left(s_{0}, \lambda+\mu, L, u_{0}\right)\right) \\
& =-\frac{\lambda}{\lambda+\mu} \Delta F_{0, \varphi}\left(s_{0}, \lambda+\mu, L, a_{0}-\theta_{0}\right) .
\end{aligned}
$$

(ii) We have

$$
\mathrm{E}\left(\mathrm{e}^{-\mu \tau_{L}} \mathbf{1}_{\left\{\tau_{L}>T\right\}}\right)=\left(\frac{(1+\varphi) s_{0}}{L}\right)^{\theta_{0} / \sigma} \mathrm{E}^{\theta_{0}}\left(\mathbf{1}_{\left\{\tau_{L}>T\right\}}\right),
$$

where $\mathrm{P}^{\theta_{0}}$ is the $\mathrm{P}$-equivalent probability measure, such that

$$
\left.\frac{\mathrm{dP}^{\theta_{0}}}{\mathrm{dP}}\right|_{\mathcal{F}_{t}}=\mathscr{E}\left(\theta_{0} W\right)_{t}=\exp \left(\theta_{0} W_{t}-\frac{1}{2} \theta_{0}^{2} t\right)
$$

and where $\mathrm{E}^{\theta_{0}}$ is the $\mathrm{P}^{\theta_{0}}$-expectation. We can write

$$
\mathrm{E}\left(\mathrm{e}^{-\mu \tau_{L}} \mathbf{1}_{\left\{\tau_{L}>T\right\}}\right)=\left(\frac{(1+\varphi) s_{0}}{L}\right)^{\theta_{0} / \sigma} \mathrm{P}^{\theta_{0}}\left(M_{T}^{a_{0}, \theta_{0}}<y, Y_{T}^{a_{0}, \theta_{0}}<x\right),
$$

where

$$
\begin{aligned}
& Y_{t}^{a_{0}, \theta_{0}}=a_{0} t+W_{t}-\theta_{0} t, \\
& M_{t}^{a_{0}, \theta_{0}}=\sup \left(Y_{s}^{a_{0}, \theta_{0}}, 0 \leq t \leq s\right), \\
& y=\left(\frac{1}{\sigma}\right) \ln \left(\frac{L}{s_{0}}\right), \quad \text { and } \\
& x=\left(\frac{1}{\sigma}\right) \ln \left(\frac{L}{s_{0}(1+\varphi)}\right) .
\end{aligned}
$$


Therefore, we obtain

$$
\mathrm{E}\left(\mathrm{e}^{-\mu \tau_{L}} \mathbf{1}_{\left\{\tau_{L}>T\right\}}\right)=\left(\frac{(1+\varphi) s_{0}}{L}\right)^{\theta_{0} / \sigma} F_{\varphi}\left(s_{0}, \lambda, L, a_{0}\right) .
$$

(iii) The third assertion follows directly from

$$
\mathrm{E}\left(\mathrm{e}^{-\mu \tau_{L}} \mathbf{1}_{\left\{\tau_{L}<T\right\}}\right)=\left(\frac{s_{0}}{L}\right)^{\theta_{0} / \sigma} \mathrm{E}^{\theta_{0}}\left(\mathbf{1}_{\left\{\tau_{L}<T\right\}}\right)=\left(\frac{s_{0}}{L}\right)^{\theta_{0} / \sigma}\left(1-F_{0}\left(s_{0}, \lambda, L, a_{0}\right)\right) .
$$

(iv) We now consider $c\left(s_{0}, L\right)=\mathrm{E}\left[\mathrm{e}^{-\mu \tau_{L}}\left(S_{\tau_{L}}-1\right)\right]$. Let us first study the case where the jump size is a positive constant $\varphi$. We have

$$
\mathrm{E}\left(S_{\tau_{L}} \mathrm{e}^{-\mu \tau_{L}} \mathbf{1}_{\left\{\tau_{L}=T\right\}}\right)=\frac{\lambda s_{0}(1+\varphi)}{\lambda+\mu-\alpha} \mathrm{E}^{\psi_{2}}\left(\mathbf{1}_{\left\{\tau_{L}=T\right\}}\right),
$$

where $\psi_{2}=(\mu-\alpha) / \lambda$ and $\mathrm{P}^{\psi_{2}}$ is the probability measure defined by $\mathrm{dP}^{\psi_{2}} /\left.\mathrm{dP}^{\sigma}\right|_{\mathcal{F}_{t}}=$ $\varepsilon(\sigma W)_{t} \&\left(\psi_{2} M\right)_{t}$. Then we obtain

$$
\begin{aligned}
\mathrm{E}\left(S_{\tau_{L}} \mathrm{e}^{-\mu \tau_{L}} \mathbf{1}_{\left\{\tau_{L}=T\right\}}\right)= & \frac{s_{0}(1+\varphi) \lambda}{\lambda+\mu-\alpha} F_{0}\left(s_{0}, \lambda+\mu-\alpha, L, v_{0}\right) \\
& -\frac{s_{0}(1+\varphi) \lambda}{\lambda+\mu-\alpha} F_{\varphi}\left(s_{0}, \lambda+\mu-\alpha, L, v_{0}\right),
\end{aligned}
$$

where $v_{0}$ is defined by (8). We conclude the proof by using (i), (ii), (iii), and the equality $c\left(s_{0}, L\right)=(L-1) \mathrm{E}\left(\mathrm{e}^{-\mu \tau_{L}} \mathbf{1}_{\left\{\tau_{L}<T\right\}}\right)+(L-1) \mathrm{E}\left(\mathrm{e}^{-\mu \tau_{L}} \mathbf{1}_{\left\{\tau_{L}>T\right\}}\right)+\mathrm{E}\left(\mathrm{e}^{-\mu \tau_{L}}\left(S_{\tau_{L}}-1\right) \mathbf{1}_{\left\{\tau_{L}=T\right\}}\right)$.

Lemma 3. Let $L$ be a frontier such that $L \geq 1$ and $L>s_{0}$. For any $\varphi$ in $]-1,0[$, we have

$$
\mathrm{E}\left(\mathrm{e}^{-\mu \tau_{L}} \mathbf{1}_{\left\{\tau_{L}>T\right\}}\right)=\left(\frac{(1+\varphi) s_{0}}{L}\right)^{\theta_{0} / \sigma} F_{0}\left(s_{0}, \lambda, L, a_{0}\right),
$$

where $a_{0}, \theta_{0}$, and $F_{0}\left(s_{0}, \lambda, L, a\right)$ are defined by (6), (7), and (4) respectively.

Proof. For any $\varphi$ in $]-1,0[$, we can write

$$
\mathrm{E}\left(\mathrm{e}^{-\mu \tau_{L}} \mathbf{1}_{\left\{\tau_{L}>T\right\}}\right)=\left(\frac{(1+\varphi) s_{0}}{L}\right)^{\theta_{0} / \sigma} \mathrm{E}^{\theta_{0}}\left(\mathbf{1}_{\left\{\tau_{L}>T\right\}}\right) .
$$

In this case we have

$$
\mathrm{E}^{\theta_{0}}\left(\mathbf{1}_{\left\{\tau_{L}>T\right\}}\right)=F_{0}\left(s_{0}, \lambda, L, a_{0}\right)
$$

\section{Acknowledgement}

Both authors would like to thank Monique Jeanblanc for her valuable comments and insights.

\section{References}

BARRIEU, P. AND Bellamy, N. (2005). Impact of market crises on real options. In Exotic Option Pricing under Advanced Lévy Models, eds A. Kyprianou, W. Schoutens and P. Wilmott, John Wiley, New York, pp. 149-168.

Brennan, M. J. And Schwartz, E. S. (1985). Evaluating natural resource investments. J. Business 58, $135-157$.

Chesney, M. And Jeanblanc, M. (2004). Pricing American currency options in an exponential Lévy model. Appl.

Math. Finance 11, 207-225. 
Dixit, A. K. AND PINDYCK, R. S. (1993). Investment under Uncertainty. Princeton University Press.

El Karoui, N. (1981). Les aspects probabilistes du contrôle stochastique. In Ecole d'Eté de Probabilités de Saint Flour IX-1979 (Lecture Notes Math. 876), Springer, New York, pp. 73-238.

Gerber, H. U. and ShiU, E. S. W. (1998). Pricing perpetual options for jump processes. N. Amer. Actuarial J. 2, 101-112.

JACKA, S. D. (1991). Optimal stopping and the American put. Math. Finance 1, 1-14.

Karatzas, I. And Shreve, S. E. (1998). Methods of Mathematical Finance. Springer, New York.

McDonald, R. ANd Siegel, D. (1986). The value of waiting to invest. Quart. J. Econom. 101, 707-727.

Merton, R. C. (1992). Continuous-Time Finance. Blackwell, Cambridge.

MoRdecKi, E. (2002). Optimal stopping and perpetual options for Lévy processes. Finance Stoch. 6, 473-493.

MuroI, Y. (2002). Pricing American put options on defaultable bonds. Asia-Pacific Financial Markets 9, $217-239$.

Peskir, G. AND Shiryaev, A. N. (2006). Optimal Stopping and Free-Boundary Problems. Birkhäuser, Basel. 